MIGRACIONES INTERNACIONALES, VOL. 12, NOTA CRÍTICA, 2021 e-ISSN 2594-0279 https://doi.org/10.33679/rmi.v1i1.2600

\title{
Jorge A. Bustamante, su contribución a los estudios sobre migraciones y fronteras
}

Jorge A. Bustamante, His Contribution to Migration and Border Studies

\section{Laura Velasco Ortiz ${ }^{1}$}

Fecha de recepción: 22 de abril de 2021

Fecha de aceptación: 28 de junio de 2021

Fecha de publicación web: 30 de diciembre de 2021

Jorge Bustamante fundó el primer Centro de Estudios Fronterizos de América Latina en $1982 .^{2} \mathrm{Su}$ legado es tangible en una consolidada institución de investigación, con sedes en seis ciudades de la frontera mexicana y una amplia presencia en el ámbito académico nacional e internacional. Sin embargo, estas notas están orientadas a destacar su contribución intelectual a los estudios sobre migraciones y fronteras. ${ }^{3}$

En los años sesenta y setenta del siglo XX, en América Latina la sociología de la migración se debatió entre las perspectivas neoclásica (Todaro, 1969), histórica estructural (Singer, 1972), y de la modernización (Germani, 1969), abocadas principalmente al estudio de las migraciones masivas del campo a la ciudad en el marco de la industrialización y de los cambios sociales y culturales que marcaron la urbanización latinoamericana. La huella de esta producción en el estudio de la migración internacional es visible, entre otros aspectos, en las unidades de análisis privilegiadas. -tales como la familia y la comunidad local-, así como en los marcos analíticos de orden nacional, otorgando un peso importante a los programas de ajuste económico que afectaron a las sociedades campesinas.

Por su temprano inicio, México es una excepción en la historia de la emigración internacional en los países latinoamericanos. En la primera parte del siglo XX ya existían los estudios pioneros de Manuel Gamio y Paul Taylor. Y para la década de los setenta la migración indocumentada había cobrado la atención en el marco de las relaciones entre México y Estados Unidos, por estudiosos como Alba-Hernández (1976), Cornelius, (1978), Ojeda (1978), y el propio Jorge Bustamante

\footnotetext{
${ }^{1}$ Colegio de la Frontera Norte, México, lvelasco@colef.mx, https://orcid.org/0000-0001-5754$\underline{6395}$

${ }^{2}$ El Centro de Estudios Fronterizos del Norte de México (Cefnomex), cambió su nombre a El Colegio de la Frontera Norte en 1986.

${ }^{3}$ Estas notas se basan en Velasco (2021).
} 
$(1975,1978) .{ }^{4}$ Con estos antecedentes, el campo de estudio de la migración mexicana a Estados Unidos se desarrolló plenamente en la década de los ochenta alimentado por diversas disciplinas, entre ellas la sociología y la antropología. Un ejemplo clásico de la interdisciplina es el libro de Return to Aztlán: The social process of international migration from Western Mexico, de Massey, Alarcón, Durand y González (1987), que presentó la perspectiva teórica de la acumulación causal y develó el papel de las redes sociales como mecanismo de reproducción de la migración a lo largo del tiempo.

Los estudios realizados por el equipo de Massey et al. (1987) combinan los enfoques antropológico y sociológico para estudiar las comunidades de origen, y observar las consecuencias de los tratados laborales entre países en la creación de redes sociales de acumulación de capitales y el efecto de las políticas migratorias en la ampliación de tales redes por los migrantes sin documentos (Massey et al. 1987). Esta obra tuvo un impacto importante en los estudios migratorios en México durante la década de los noventa, colocando a las redes de migrantes como el mecanismo más importante de la reproducción de la migración, alejándose de los estudios pushpull en la migración internacional.

Sin embargo, como señaló Zolberg (1999), en la década de los noventa la literatura sobre migración era escéptica sobre la efectividad de las políticas migratorias en las formas y dinámicas de los flujos migratorios, en particular para los que provenían de países con fronteras continuas, como el caso de México. En este contexto, el trabajo de Bustamante (2000) retomó diversos elementos de la historia migratoria y fronteriza entre México y Estados Unidos, entre ellos la temprana racialización de los inmigrantes mexicanos (Zolberg, 1999; Andreas, 2003; Alonso, 1994), dada la anexión de territorio mexicano durante el siglo XIX y la temprana ilegalización de la migración mexicana, contradictoriamente surgida del propio programa de braceros, y que dio origen a la expresión "wetbacks" (Ngai, 2005).

Al observar los efectos de las políticas migratorias de Estados Unidos en el orden social migratorio mexicano, la contribución de Bustamante $(1989)^{5}$ se extiende a la conceptualización de la región fronteriza entre ambos países como espacio adyacente y asimétrico, pero de alta interacción social. Tal conceptualización refleja la influencia intelectual de Mario Ojeda (1978), quien acuñó tal visión de desigualdad vecinal al caracterizar la estructura de las relaciones entre México y Estados Unidos.

\footnotetext{
${ }^{4}$ En los años setenta, El Colegio de México fungió como un espacio intelectual importante para la gestación del campo de estudios sobre la migración indocumentada desde México a Estados Unidos, a través de su revista Foro Internacional.

${ }^{5}$ Los temas fronterizos empezaban a cobrar interés académico a fines de los años setenta, como se puede constatar en la reseña que hace el propio Bustamante (1989, pp. 7-24) del Primer Simposio Nacional sobre Estudio Fronterizos, organizado por El Colegio de México y celebrado en Monterrey en 1979. Para entonces, Bustamante (1975) había publicado ya Espaldas mojadas: Materia prima para la expansión del capital norteamericano.
} 
El cierre de los programas laborales en la década de los sesenta y las consecuentes expulsiones de trabajadores contratados temporalmente, ${ }^{6}$ dieron lugar a una oleada de estudios que centraron su interés en las consecuencias sociales del retorno de los migrantes mexicanos, y llamaron la atención sobre la urgencia de inversiones que permitieran mitigar el creciente desempleo en la región fronteriza (Fernández, 1980), y de estudiar la frontera norte de México (Bustamante, 1978).

En la década de los ochenta los estudios fronterizos mexicanos pronto se enfocaron en tres líneas de indagación: a) los impactos de la migración en la tardía urbanización e industrialización de las regiones fronterizas (Fernández-Kelly 1984; Alegría 1989), b) la emergencia de una cultura fronteriza ligada a la interacción transfronteriza y a la inmigración atraída por la pujante industria maquiladora (Iglesias, 1985;Valenzuela, 1988; García, 1990), con gran influencia de la producción académica de los estudiosos chicanos (Vélez- Ibáñez, 1996), y c) el estudio de la frontera como espacio de circulación y cruce sin documentos (Bustamante, 1989, 2000).

Los aportes de Jorge Bustamante se inscriben en esas tres líneas, con derivaciones que atañen tanto a a los estudios fronterizos como a los de las migraciones internacionales. En relación con la conceptualización de la frontera, existía una polémica implícita sobre su definición como una región binacional por su adyacencia, o bien transfronteriza, debido a su interacción. Al respecto, adoptando un enfoque weberiano, Bustamante acuñó el concepto de frontera como espacio relacional con interacciones transfronterizas de distinto orden, marcadas por la asimetría (Bustamante, 1989), dado que respondía a las lógicas nacionales respectivas a la vez que a las relaciones empíricas regionalmente localizadas entre ambos países. ${ }^{7}$

En relación con la migración, mientras los estudios vigentes en la década de los ochenta y parte de los noventa se enfocaban en las localidades de origen o destino, ${ }^{8}$ Bustamante se enfocó en el cruce cladestino, la movilidad circular y el control fronterizo por parte de Estados Unidos. Este planteamiento caracterizó la producción intelectual de Bustamante y sentó las bases para la comprensión de la tensión entre la fluidez de las interacciones cotidianas fronterizas, con un fuerte impacto en la cultura, y por otra parte, en el cierre de la frontera para los migrantes pobres sin documentos. En esta segunda línea, el cruce clandestino, la circulación y el control estatal fueron vistos como procesos constitutivos de la frontera misma.

El foco en el fenómeno de los cruces clandestinos y la movilidad circular dio origen a una visión novedosa de las fronteras geopolíticas como espacios de asimetría y desigualdad (Ojeda, 1978; Bustamante, 2000), a consecuencia de niveles de desarrollo económico asimétrico, cuyos principales indicadores fueron el diferencial salarial y el control unilateral de la frontera por parte

${ }^{6}$ El programa de Braceros funcionó de 1942 a 1964 a través de la contratación de trabajadores mexicanos para trabajar en la agricultura, principalmente.

${ }^{7} \mathrm{Al}$ respecto, ver Alegría (1989), quien demostró que las dinámicas fronterizas respondían más a las respectivas dinámicas nacionales que a las de una región integrada, por lo que dominaba más la asimetría que la interacción regional en la estructuración urbana fronteriza.

${ }^{8}$ Simultáneamente, iniciaba la reflexión sobre las consecuencias de la amnistía de 1986 (IRCA) en la migración por reunificación familiar. 
de Estados Unidos. El estudio de los controles sobre los cruces fronterizos, así como las dinámicas, magnitudes y condiciones de cruce, se convirtieron en un programa de investigación con una metodología novedosa a nivel continental para el estudio de los flujos migratorios, primero con los migrantes indocumentados, y posteriormente, ampliado a potenciales cruzadores y migrantes de retorno.

En 1987 Bustamante creó el Proyecto Cañón Zapata, ${ }^{9}$ cuyo objetivo fue calcular el número de cruces de migrantes hacia Estados Unidos mediante una técnica bastante original, basada en el registro fotográfico desde una colina de la ciudad de Tijuana (Bustamante, 2000), mientras que de forma paralela se realizaban entrevistas en los sitios de cruce más comunes en varias ciudades fronterizas con Estados Unidos. Por primera vez la relación entre el cruce indocumentado y los controles fronterizos se volvió un tema de investigación sistemática en México, y se inició la distinción de otras movilidades transfronterizas irregulares como los commuters sin visas de trabajo o visa abusers (Bustamante, 2000, p. 18). Este proyecto fue la semilla de la actual Encuesta sobre Migración en la Frontera Norte (EMIF Norte), que data de 1993. A través de una muestra probabilística representativa en espacio y tiempo, la EMIF constituye, a nivel continental, el proyecto más ambicioso sobre la medición y caracterización de flujos migratorios internacionales.

A la fecha, la EMIF cuenta con una batería de bases de datos de casi tres décadas, incluyendo flujos migratorios de sur norte y norte sur en la frontera México-Estados Unidos. A partir de 2004, se amplió a la frontera México-Guatemala (EMIF Sur). A lo largo del tiempo, el programa de investigación sobre flujos migratorios, dio pie a importantes políticas públicas. El proyecto Cañón Zapata contribuyó a la creación del Programa Paisano y el Grupo Beta ${ }^{10}$ para la protección de los migrantes en el cruce ${ }^{11}$. Posteriormente, el papel de Jorge Bustamante como Relator especial sobre los derechos humanos de los migrantes de la ONU (2005-2011), le permitió visibilizar a nivel

\footnotetext{
${ }^{9}$ Nombre del lugar por donde cruzaba un gran número de personas sin documentos desde la ciudad de Tijuana, ya que en esa época no existía el muro fronterizo. La realización de entrevistas in situ se extendió hasta 1998 (Bustamante, 2000).

${ }^{10}$ El camino de cómo el conocimiento generado por la investigación se traduce en política pública escapa a estas notas sobre el legado intelectual de Jorge A. Bustamante en el campo de los estudios sobre migración y fronteras. Sin embargo, es posible señalar de acuerdo con la entrevista que el mismo Bustamante otorgó a un corresponsal de la revista Proceso (Ponce, 2012), su estrategia siguió tres pasos: investigar con rigor científico, divulgar los resultados de investigación en foros amplios, y realizar una gestión constante a nivel federal para la creación de ambos programas. En particular, ver la experiencia del Grupo Beta, el cual inició como un programa piloto de protección a los migrantes en Baja California en 1990 (Weiss y López, 2011), después de las gestiones con el gobierno federal de Bustamante, tras documentar los abusos que sufrían los migrantes en el trayecto y el cruce fronterizo de lado mexicano. La divulgación de tales hallazgos trajo tensión y riesgos para su persona y para El Colef, tal como lo narra en la entrevista antes mencionada (Ponce, 2012).

${ }^{11}$ Agradezco a María Eugenia Anguiano la precisión de este dato.
} 
internacional la vulnerabilidad de los migrantes sin documentos, adultos y menores de edad, con base en evidencia científica y avizorar las crisis humanitarias que hoy atestiguamos en el mundo.

Pero más allá de las diversas consecuencias puntuales de política, la medición y caracterización de los flujos migratorios a partir de la década de los noventa permitió contar con datos confiables, producidos con una metodología científica por un centro de investigación mexicano, y no depender de los datos del entonces Servicio de Inmigración y Naturalización (INS) para el registro sistemático de procesos que son cruciales para México.

Más de tres décadas después, los aportes de Jorge Bustamante contribuyen a clarificar la relación de los actuales regímenes de control migratorio y fronterizo con las dinámicas y condiciones de las movilidades humanas, cada vez más complejas y diversas frente al incremento de las desigualdades, la violencia y las crisis políticas en el continente. Jorge Bustamante influyó la política migratoria desde la academia, reivindicando constantemente la importancia y el papel de la ciencia para brindar conocimientos específicos y confiables. Además de sus aportes conceptuales, la construcción de las EMIF constituye un legado que va más allá de la propia institución y que pertenece a los logros de la ciencia en México.

\section{REFERENCIAS}

Alba-Hernández, F. (1976). Exodo silencioso: la emigración de trabajadores mexicanos a Estados Unidos. Foro Internacional, 17(66), 152-179. Recuperado de https://forointernacional.colmex.mx/index.php/fi/article/view/723

Alegría, T. (1989). La ciudad y los procesos trasfronterizos entre México y Estados Unidos. Frontera Norte, 1(2), 53-90. doi: https://doi.org/10.17428/rfn.v1i2.1654

Alonso, A. M. (1994). The politics of space, time and substance: State formation, nationalism and ethnicity. Annual Review of Anthropology, 23, 379-405. doi: https://doi.org/10.1146/annurev.an.23.100194.002115

Andreas, P. (2003). A tale of two borders: The US-Mexico and US-Canada lines after 9/11. The Center for Comparative Immigration Studies. Working Paper, (77). Recuperado de https://ccis.ucsd.edu/ files/wp77.pdf

Bustamante, J. (1975). Espaldas mojadas: Materia prima para la expansión del capital norteamericano. México: El Colegio de México.

Bustamante, J. (1978). Emigración indocumentada a los Estados Unidos. Foro Internacional, 18(3), 430-463. Recuperado de https://forointernacional.colmex.mx/index.php/fi/article/view/796

Bustamante, J. (1989). Frontera México-Estados Unidos: reflexiones para un marco teórico. Frontera Norte, 1(1), 7-24. doi: https://doi.org/10.17428/rfn.v1i1.1666 
Bustamante, J. (2000). Migración irregular de México a Estados Unidos: Diez años de investigación del Proyecto Cañón Zapata. Frontera Norte, 12(23), 7-49. doi: https://doi.org/10.17428/rfn.v12i23.1393

Cornelius, W. (1978). La migración ilegal mexicana a los Estados Unidos: conclusiones de investigación recientes, implicaciones políticas y prioridades de investigación. Foro Internacional, $\quad$ 18(3), 420-429. $\quad$ Recuperado de https://forointernacional.colmex.mx/index.php/fi/article/view/795

Fernández, R. (1980). La frontera México-Estados Unidos. Ciudad de México: Terra Nova.

Fernandez-Kelly, M. P. (1984). For we are sold, I and my people: Women and industry in Mexico's frontier. Albany: State University of New York Press.

García Canclini, N. (1990). Culturas hibridas. Estrategias para salir y entrar de la modernidad. México: Grijalbo.

Germani, G. (1969). Sociología de la Modernización. Estudios teóricos, metodológicos y aplicados a América Latina. Buenos Aires: Paidós.

Iglesias, N. (1985). La flor más bella de la maquiladora. Ciudad de México: Secretaría de Educación Pública/Centro de Estudios Fronerizos.

Massey, D., Alarcón, R., Durand, J. y González, H. (1987). Return to Aztlan: The social process of international migration from Western Mexico. Berkeley: University of California Press.

Ngai, M. M. (2005). Impossible subjects: Illegal aliens and the making of modern America. Estados Unidos: Princeton University Press.

Ojeda, M. (1978) Cuestiones claves en las relaciones México-Estados Unidos. Foro Internacional, $19(2)$ 304-310. Recuperado de https://forointernacional.colmex.mx/index.php/fi/article/view/2348

Ponce, R. (21 de agosto de 2012). Bustamante: De la frontera norte a las fronteras del mundo. Revista Proceso. Recuperado de https://www.proceso.com.mx/reportajes/2012/8/21/bustamante-de-la-frontera-norte-lasfronteras-del-mundo-107343.html

Singer, P. (1972). Migraciones internas. Consideraciones teóricas sobre su estudio. En H. Muñoz, O. de Oliveira, P. Singer y C. Stern (eds.), Migración y desarrollo. Consideraciones teóricas, (pp. 45-68). Buenos Aires: Consejo Latinoamericano de Ciencias Sociales.

Todaro, M. (1969). A model of labor migration and urban unemployment in less developed countries. American Economic Review, 59(1), 138-148. Recuperado de https://www.jstor.org/stable/1811100

Valenzuela, J. M. (1988). ¡A la brava ése! Identidades juveniles en México: cholos, punks y chavos-banda. Tijuana: El Colegio de la Frontera Norte/UNAM. 
Velasco, L. (2021). Migration, Borders and Identity. En X. Bada y L. Rivera Sánchez (Eds.), The Oxford Handbook of Sociology of Latin America, (pp. 449-464). Oxford University Press. Doi:10.1093/oxfordhb/9780190926557.013.28

Vélez-Ibáñez, C. (1996). Border visions: Mexican cultures of the Southwest United states. Tucson: University of Arizona Press.

Weiss, L. y López, P. (2011). México: políticas públicas beneficiando a los migrantes. México: OIM. Recuperado de https://publications.iom.int/system/files/pdf/oim_pp_sp.pdf

Zolberg, A. R. (1999). Matters of state: Theorizing immigration policy. En C. Hirschman, P. Kasinitz y J. De Wind (Eds.), The handbook of international migration: The American experience, (pp. 71-93). Nueva York: Russell Sage Foundation. 